\title{
Dual payoff band control of reaction time*
}

\author{
CAROL A. SASLOW \\ Oregon State University, Conallis. Oregon 97331
}

\begin{abstract}
The location of a S's simple reaction time (RT) distribution can be controlled by differential reinforcement of RTs that fall within specified temporal limits called a payoff band. Both humans and monkeys can gradually shift the location of a single RT distribution over hundreds of milliseconds in accord with changing payoff band requirements. This study establishes that trained human Ss can also accurately shift their response latencies back and forth between two different RT distributions when the payoff band is changed from trial to trial. On each trial, the color of the waming stimulus indicated which of two payoff bands would be in effect when the $S$ reacted to a light flashed $1,500 \mathrm{msec}$ later. The RT distributions produced under the condition of random trial-to-trial switching between two payoff bands were the same as the low-variability RT distributions produced when only one payoff band was in effect over a long series of trials.
\end{abstract}

Interest in simple reaction time (RT) has generally been centered around the effects of stimulus variables (Froeberg, 1907: Cocholle, 1940; Lit, Young, \& Shaffer, 1971). The usual assumption has been that instructions to respond quickly but accurately produce, in a human $S$, a consistent bias for speed which ensures an asymptotic performance under all stimulus conditions. However. there is considerable experimental evidence indicating that $S$ expectations and trial-to-trial information feedback and payoff contingencies are extremely important in determining RT to a given stimulus (Johanson, 1922; Thrane, 1961, 1962; Gordon, 1967; Snodgrass, Luce, \& Galanter, 1967; Snodgrass, 1969: Saslow, 1968, 1972). Because of this evidence, it seems that a more appropriate term for all such timing distributions would be "response latency" rather than the traditional "reaction time," which carries the implication that the $S$ is always producing stimulus-driven minimal delays independent of motivational and other factors. In the present paper, the terms will be used interchangeably and will be operationally defined as referring to the measured interval between stimulus and response onsets.

Snodgrass et al (1967) developed the payoff band technique for control of RT by feedback and reinforcement. Using this technique, a $S$ is reinforced for a particular set of response latencies and not reinforced, or penalized, for others. For example, in an auditory RT task a S might be placed on a 144-164-msec payoff band. He would then be reinforced only on those trials when his RT fell within the specified limits, and not reinforced if his RT was less than 144 or greater than $164 \mathrm{msec}$. Research employing the payoff-band technique, for both man (Snodgrass et al, 1967) and monkey (Saslow, 1968), has established that the mean or median of the response latency distribution can be continuously shifted over a wide range of delays by

*This research was partly supported by a general research grant from the Oregon State University Graduate School and an unsponsored research grant from the Oregon State University Computer Center. changing payoff-band location. The variability of the latency distribution remains a fixed proportion of the mean or median, and, for the well-trained $S$, the standard deviation of a time estimation or reaction time distribution should remain about $10 \%$ of the mean (Chocholle, 1940; Snodgrass et al, 1967; Saslow, 1968, 1972).

Based on this data, Snodgrass et al proposed a model that states that any RT distribution is potentially a mixture, in varying proportions, of time estimations from the warning stimulus, true reactions and time estimations from the reaction stimulus. Extensive research (Saslow, 1968, 1972; Snodgrass, 1969) indicates that the proportions of the two types of time estimation included in an RT distribution depend on the location of the payoff band and can be detected by examination of the variability of the RT distribution. When a payoff band is moved closer in time to the reaction stimulus, both the average $\mathrm{RT}$ and variability decrease until the $S$ starts including foreperiod time estimations in an attempt to continue to meet band requirements. Inclusion of foreperiod time estimations rapidly inflates the variability of the RT distribution.

Having found that both human and monkey Ss are capable of exercising very precise control over the location of a single response latency distribution in accord with temporal reinforcement contingencies, it is of interest whether or not the $S$ can be accurately programmed for two different delays at the same time and still produce appropriate latency distributions of low variability. Snodgrass et al (1967), in one series of experiments, reinforced the $S$ for response latencies falling within a $100-200-\mathrm{msec}$ band when the reaction stimulus was a $1,000 \cdot \mathrm{Hz}$ tone, but reinforced only latencies falling in a band of $200-300 \mathrm{msec}$ when the reaction stimulus was a $1,200-\mathrm{Hz}$ tone. The two reaction stimuli were randomly varied from trial to trial, and only a single response was used. The Ss did not produce two separate latency distributions appropriate to the two different bands. Instead, they produced a single latency distribution for both stimuli with a mean RT 
Table 1

Total Number of Trials on Fast and Slow Bands Before the First Test on Random Switching of Bands

\begin{tabular}{|c|c|c|c|c|c|c|}
\hline & \multicolumn{2}{|c|}{ S T.B. } & \multicolumn{2}{|c|}{ S C.S. } & \multicolumn{2}{|c|}{ S B.M. } \\
\hline & Fast & Slow & Fast & Slow & Fast & Slow \\
\hline $\begin{array}{l}\text { 1. Training-Reduction of bandwidth to } 50 \mathrm{msec} \\
\text { 2. Slow band only in } 100 \text {-trial blocks } \\
\text { 3. 100-trial blocks on Fast or Slow } \\
\text { 4. Band alternated at reinforcement }\end{array}$ & $\begin{array}{r}-- \\
1500 \\
817\end{array}$ & $\begin{array}{r}2300 \\
800 \\
2500 \\
955\end{array}$ & $\begin{array}{l}-- \\
-- \\
300 \\
300\end{array}$ & $\begin{array}{r}2300 \\
300 \\
500 \\
385\end{array}$ & $\begin{array}{l}-- \\
-\overline{-} \\
234\end{array}$ & $\begin{array}{r}2700 \\
1600 \\
500 \\
294\end{array}$ \\
\hline Total & 2317 & 6555 & 600 & 3485 & 734 & 5094 \\
\hline
\end{tabular}

appropriate to the slower band and with an inflated standard deviation which was $25 \%$ of the mean RT. These results were similar to those gotten in a choice RT design involving two responses, each with its own payoff band.

The present study was designed to determine if a human $S$ could be trained to produce two different precise delays in the same simple RT task when recognition of the nature of the reaction stimulus before responding was not required. Accordingly, the same reaction stimulus was used on all trials, and the color of the warning stimulus was the cue which indicated which of two payoff bands would be in effect on a given trial. It was predicted that under these circumstances a trained $S$ could accurately switch back and forth between two delays and produce two separate latency distributions, each appropriate to its respective band. It was expected, however, that these two latency distributions would be more variable than those obtained on the same payoff bands when only one band at a time was in effect over a large block of trials.

\section{METHOD}

\section{Subjects}

Three adults served as paid Ss. One (C.S.) had had previous experience as a $S$ in simple and choice reaction time experiments. The others (T.B., B.M.) were naive.

\section{Apparatus}

The stimulus display consisted of two dim incandescent warning lights, a red one on the left and a green one on the right. Centered below these was a neon bulb (NE-76) used as the reaction stimulus. Below the reaction stimulus was a white incandescent feedback light.

Timing of trial events was controlled by a system of six timers (Tektronix 162) and a network of fast-acting relays (Millisec). The control system was isolated from the $S$ to avoid auditory cues.

Response latencies for release of a telegraph key to onset of the neon light were recorded accurate to the nearest millisecond by a Hewlett-Packard counter.

\section{Trial Events}

The $\mathrm{S}$ pressed and held down a telegraph key with his right forefinger to start a trial. After $500 \mathrm{msec}$, either the red or the green warning light came on. There was a fixed foreperiod of $1,500 \mathrm{msec}$ between onset of the warning light and onset of a $10-\mathrm{msec}$ flash of the neon reaction stimulus. The $S$ was instructed to release the key after the reaction stimulus. Key release terminated the warning stimulus. If the latency of the key release fell within specified time limits, the feedback light came on for $500 \mathrm{msec}$ and the $\mathrm{S}$ had 1 cent added to his account. If the latency was too long or too short, neither feedback nor reinforcement was given, and the $S$ initiated a new trial when he was ready. Anticipations, which occurred infrequently and only in the initial stages of training, also terminated a trial without reinforcement.

A block of 100 trials constituted a run and took from 10 to $15 \mathrm{~min}$ to complete.

\section{Training on Fast and Slow Bands}

The course of training is summarized separately for each $\mathrm{S}$ in Table 1. Initially, a $S$ was placed on a pay off band with limits of 275 and $375 \mathrm{msec}$, and each trial was signaled by the red warning light. Releases with latencies less than $275 \mathrm{msec}$ or greater than $375 \mathrm{msec}$ were not reinforced. As soon as the $\mathrm{S}$ was receiving reinforcement on $60 \%$ to $70 \%$ of the trials in a run, the width of the band was reduced by $10 \mathrm{msec}$. This continued until the $S$ was working with a band of $300-350 \mathrm{msec}$. Training was then continued on this "slow" band until the S was earning reinforcement on at least $65 \%$ of the trials, had reduced the standard deviation of his response latency distribution for each run to $15 \%$ or less of the mean latency, and was producing distributions from run to run that looked highly similar.

Once the S's performance stabilized on the slow band, alternate runs were introduced on a "fast" payoff band. On these runs, the green warning light was used and the payoff band limits were 175 and $225 \mathrm{msec}$. Prolonged training was not given on this fast band, since this range of times approximated the latencies that the Ss first produced when instructed to go "as fast as possible without anticipating." Runs on the fast (green warning) and slow (red warning) bands were alternated until the $\mathrm{S}$ was producing stable latency distributions appropriate to each band.

In the final phase of training, the payoff band, along with its appropriate warning stimulus, was changed following each reinforcement within a run. Thus, a $S$ who started a run on the fast band would be shifted to the slow band as soon as he had produced a latency within the fast band. When he achieved reinforcement on the slow-band contingency, he would be shifted back to the fast band for the next trial, and so on. To maintain stability on both the fast and slow bands, fast-band and slow-band blocked runs with 100 trials on only one band at a time were interspersed with the "switch-on-reinforcement" runs.

\section{Switching at Random Between Fast and Slow Bands}

Within test runs, the two bands were switched randomly from trial to trial. On each trial, the color of the warning stimulus was the first cue the $S$ had as to whether he should be trying to meet the fast-band $(175-225 \mathrm{msec})$ or slow-band $(300-350 \mathrm{msec})$ contingencies. A test run was 200 trials long and contained 100 trials on each of the two bands. In order to prevent long sequences of trials on the same band, randomization was done over blocks of 20 trials. 
Table 2

Comparison of Response Latencies from Blocked vs Random-Switch Runs for Fast $(175-225 \mathrm{msec})$ and Slow $(300-350 \mathrm{msec})$ Bands (Sample Sizes $=100)$

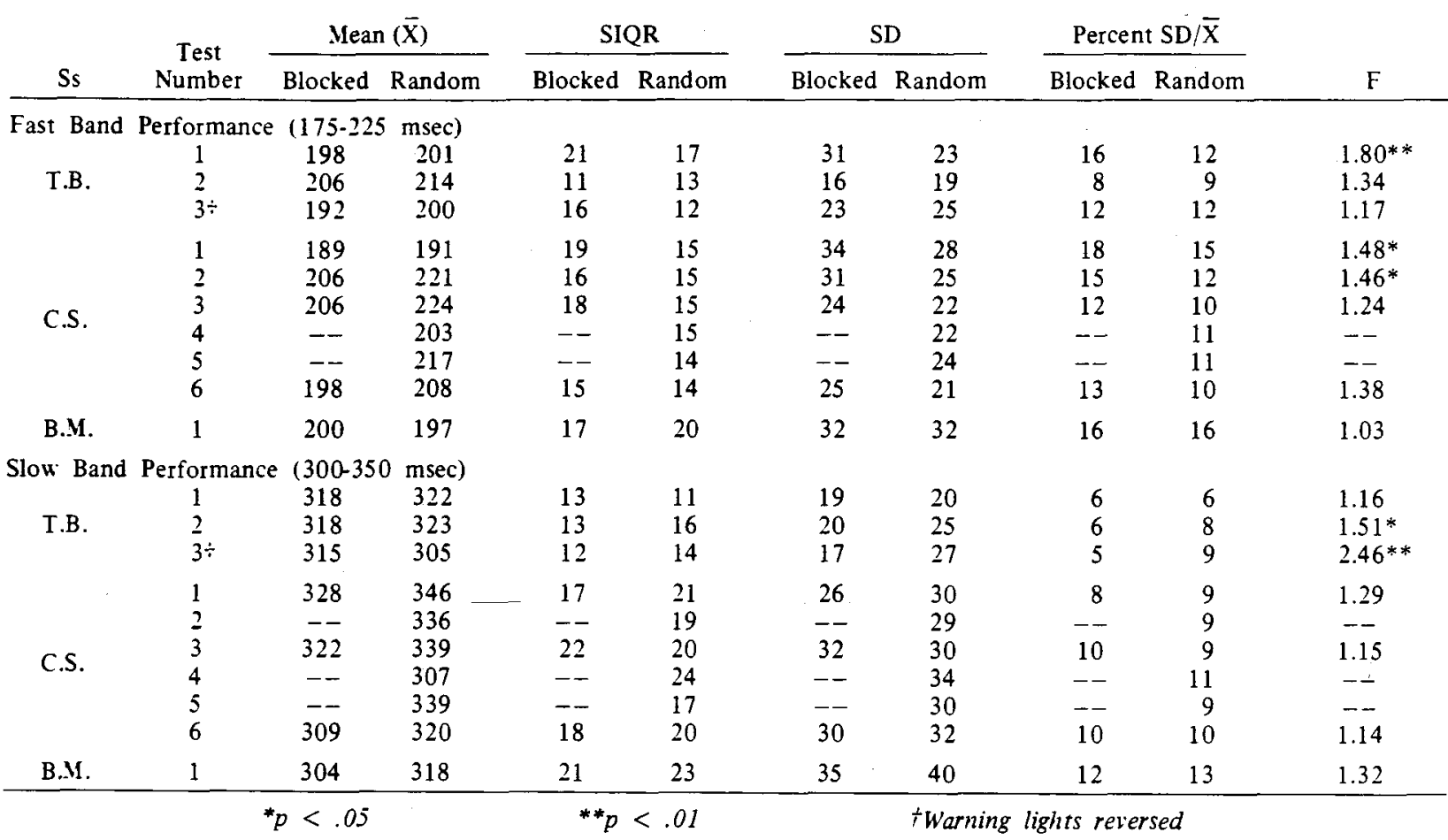

To provide comparison data, blocked runs with 100 trials on a single band were interspersed with the test runs.

\section{Reversal of Warning Lights for Fast and Slow Bands}

One S (T.B.) was retrained on the fast and slow bands with the meaning of the warning lights reversed, so that green signaled a slow-band trial and red, a fast-band trial. He resumed training under the fast band only, slow band only, and switch-at-reinforcement conditions until the fast- and slow-band latency distributions were again stable. This took 1,300 additional trials. He was then retested on random switching between the bands with the reversed warning lights.

\section{Switching Between 80-msec-wide Slow and Very Slow Bands}

Two of the Ss (C.S. and T.B.) were subsequently trained to meet an 80 -msec-wide band of $485-565 \mathrm{msec}$ signaled by the green warning light. Alternated with training runs on this "very slow" band were runs on a widened slow band (285-365 msec) paired with the red warning light.

Training was continued on the very slow band until the $S$ was producing latency distributions with the mean in the band and the standard deviation less than $15 \%$ of the mean. Then switch-on-reinforcement training runs were introduced, changing back and forth between the slow and very slow bands each time the $S$ achieved reinforcement. Finally, the $S$ was tested with random trial-to-trial switching between the two bands.

\section{RESULTS}

\section{Fast vs Slow Band}

The $S$ means, standard deviations, and semi-interquartile ranges for the switch-at-random test runs are given in Table 2. Median RTs are not included in the table, as in all cases they were within $5 \mathrm{msec}$ of the mean RTs. Statistics for latency distributions from 100-trial blocks on only one band at a time are given for comparison. In each case, the comparison blocked runs were done on the same day as the test run. Examination of the means for the fast and slow bands indicated that the Ss' latency distributions were approximately centered over the appropriate payoff band. Examination of the variability indicated that the Ss' standard deviations were generally less than $15 \%$ of the means and, in most cases, closer to $10 \%$ of the means. Semi-interquartile ranges were consistently between $5 \%$ and $10 \%$ of the median RT. Overall, the relative variability of the fast-band latencies was somewhat higher than for the slow band.

Within each band, the statistics for the blocked condition and the random condition are highly similar. The means of the latency distributions are located within the appropriate bands in all cases. Semi-interquartile ranges are nearly the same, with no indication that one condition consistently produced less variability than the other. An $F$ ratio was used to test the hypothesis that the latency distribution variances were the same whether obtained under blocked or random conditions (Freund, 1962, pp. 200-201). The F ratios were generally not significant, indicating homogeneity of variance despite the change in 


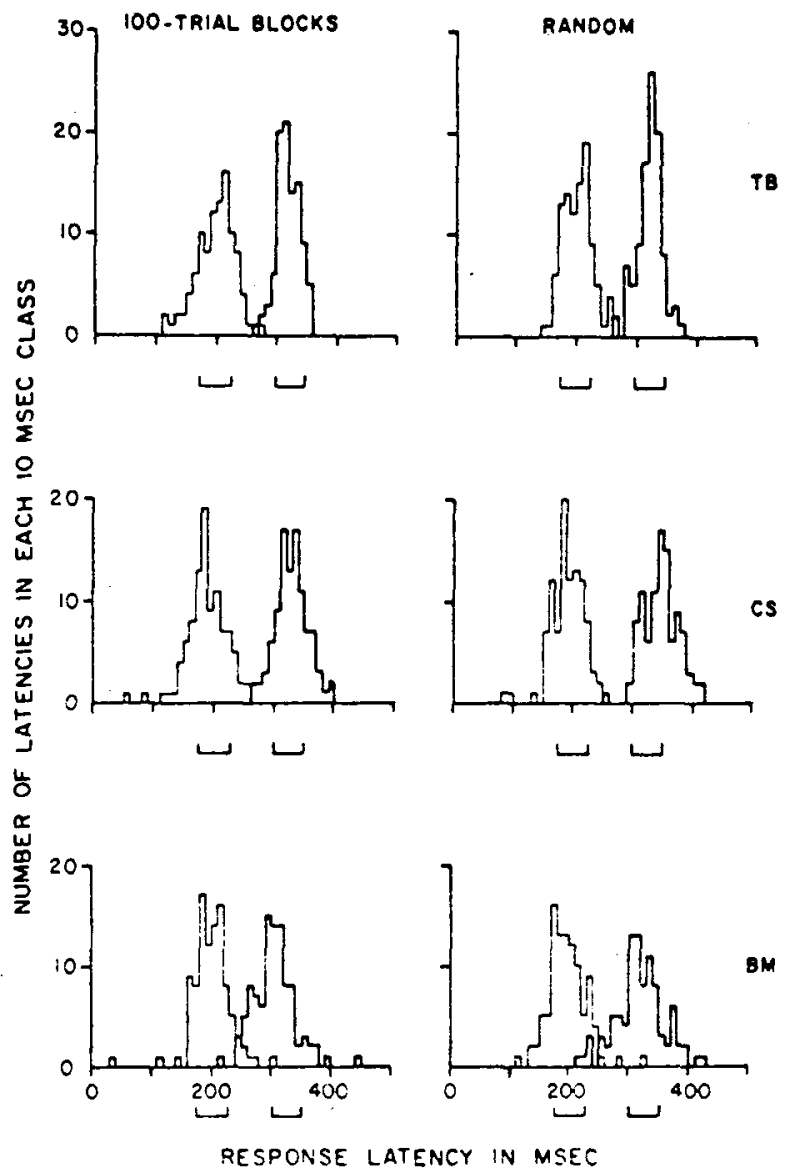

Fig. 1. Response latency histograms for runs with 50-msec-wide fast $(175-225 \mathrm{msec})$ and slow $(300-350 \mathrm{msec})$ payoff bands for three Ss. Data for the left column comes from 100-trial blocks on each band. Data for the right column comes from the first run in which the Ss were randomly switched between bands from trial to trial. Each histogram is composed of 100 latencies. Width and location of payoff bands is indicated below each histogram.

condition. For the fastest band, when the $F$ ratios were significant, it was always in the direction of less variability in latencies under the random than under the blocked conditions. For the slower band, in the two cases where the $F$ ratio was significant, there was more variability under the random condition.

The third test run for T.B., done with reversed warning stimuli, gave results similar to his other test runs.

The graphs in Fig. 1 compare the fast- and slow-band performances on the first test run for blocked and random conditions. The Ss appeared to maintain as much differentiation between their fast- and slow-latency distributions when the two types of trials were randomly intermixed for the first time as when they were blocked.

The graphs in Fig. 2 permit direct comparison of latency distributions gathered under the blocked and random conditions for each band. The data came from test runs for two Ss in which their latency distributions had very low relative variability. Inspection of the graphs indicates that while the Ss might shift their mean latency slightly between conditions, response latency distributions gathered under blocked and random conditions remained very similar.

\section{Slow vs Very Slow Band}

The results of the test runs comparing latency distributions for slow $(285.365 \mathrm{msec})$ and very slow $(485.565 \mathrm{msec})$ bands under the two conditions are given in Table 3 and Fig. 3. Again, the mean latencies were appropriate to their respective bands, and the standard deviations were about $10 \%$ of the means. The statistics for the distributions gathered under the blocked and random conditions were very similar, and three of the four $F$ ratios were not significant. One $F$ ratio was significant at the .01 level, indicating that, in this case, the S's performance under the random condition was slightly more variable than under the blocked.

Inspection of Fig. 3 confirmed that the Ss were again able to differentiate their latency distributions between two different payoff bands regardless of whether the
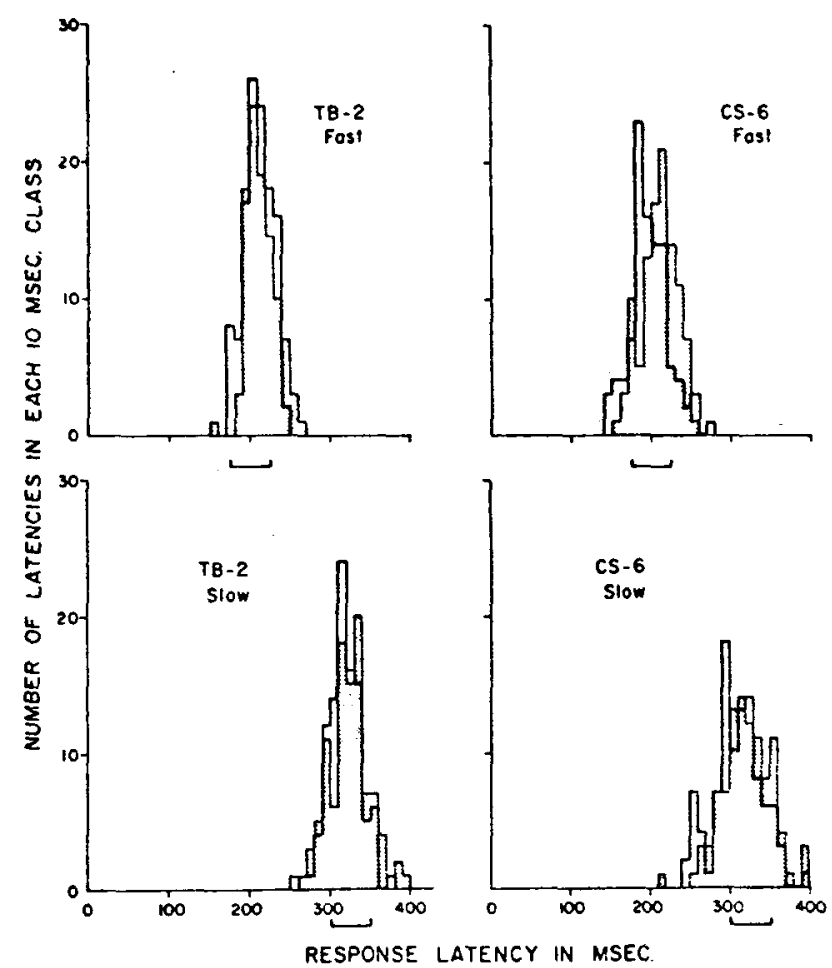

Fig. 2. Response latency histograms for runs with 50 -msec-wide fast $(175-225 \mathrm{msec})$ and slow $(300-350 \mathrm{msec})$ payoff bands for two Ss. Data comes from Test 2 for T.B. and Test 6 for C.S. The left-hand column compares latency distributions from blocked (heavy line) and random (filled histogram) conditions for the fast band. The right-hand column compares results of blocked and random conditions for the slow band. Each histogram is composed of 100 latencies. 
Table 3

Comparison of Response Latencies from Blocked vs Random-Switch Runs for Slow (285-365 msec) and Very Slow $(485-565 \mathrm{msec})$ Bands (Sample Sizes $=100$ )

\begin{tabular}{|c|c|c|c|c|c|c|c|c|c|}
\hline \multirow[b]{2}{*}{ Ss } & \multicolumn{2}{|c|}{ Mean } & \multicolumn{2}{|c|}{ SIQR } & \multicolumn{2}{|c|}{ SD } & \multicolumn{2}{|c|}{ 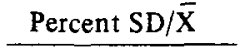 } & \multirow[b]{2}{*}{$\mathrm{F}$} \\
\hline & Blocked & Random & Blocked & Random & Blocked & Random & Blocked & Random & \\
\hline \multicolumn{10}{|c|}{ Slow Band (285-365) } \\
\hline $\begin{array}{l}\text { T.B. } \\
\text { C.S. }\end{array}$ & $\begin{array}{l}326 \\
326\end{array}$ & $\begin{array}{l}332 \\
340\end{array}$ & $\begin{array}{l}17 \\
19\end{array}$ & $\begin{array}{l}28 \\
26\end{array}$ & $\begin{array}{l}27 \\
38\end{array}$ & $\begin{array}{l}38 \\
37\end{array}$ & $\begin{array}{r}8 \\
12\end{array}$ & $\begin{array}{l}11 \\
11\end{array}$ & $\begin{array}{l}1.99 * \\
1.10\end{array}$ \\
\hline \multicolumn{10}{|c|}{ Very Slọw Band (485-565) } \\
\hline $\begin{array}{l}\text { T.B. } \\
\text { C.S. }\end{array}$ & $\begin{array}{l}506 \\
510\end{array}$ & $\begin{array}{l}492 \\
501\end{array}$ & $\begin{array}{l}24 \\
33\end{array}$ & $\begin{array}{l}22 \\
32\end{array}$ & $\begin{array}{l}36 \\
48\end{array}$ & $\begin{array}{l}36 \\
46\end{array}$ & $\begin{array}{l}7 \\
9\end{array}$ & $\begin{array}{l}7 \\
9\end{array}$ & $\begin{array}{l}1.03 \\
1.11\end{array}$ \\
\hline
\end{tabular}

trials on a given band were blocked or were randomly intermixed with trials on another band.

\section{DISCUSSION}

The Ss clearly were able to produce two separate latency distributions, even when the payoff band contingency was randomly varied from trial to trial. Contrary to expectations, these latency distributions were not consistently more variable than the distributions produced when the Ss had 100 trials in a row on a single band. These results occurred whether the Ss were switching back and forth between latencies of the order of their "natural RT" (175.225-msec band) and a moderate delay (300-350-msec band) or between two definitely delayed responses (a 285-365-msec band vs a $485-565-\mathrm{msec}$ band). The color of the warning stimulus served only as an informational cue and bore no necessary relation to the latencies themselves, as was demonstrated by the performance of the $S$ for whom the significance of the color cues was reversed with little effect on his ability to accurately switch back and forth between two latency distributions.

The characteristics of the latency distributions obtained in this experiment differ from traditional reaction time distributions, gathered without attention to motivational factors, in not having a long tail of slower reactions. Typically, both RT and time estimation distributions obtained with feedback and reinforcement are symmetrical, sharply peaked, and, if the $S$ is sufficiently trained, of low variability (Snodgrass, 1969; Saslow, 1972). Such distributions are too sharply peaked to be well fit by normal distribution approximations (Snodgrass, 1969), and for that reason comparison of the distributions on the basis of their semi-interquartile ranges rather than their variances is more appropriate, but statistics are not yet well developed for such comparisons. Visual inspection of the semi-interquartile ranges in Tables 2 and 3 shows even less evidence for a consistent difference in variability between blocked and random payoff band performance than do the standard deviations and $F$ ratios.

The variability of the latency distributions was generally low and of the order expected of well-trained
Ss in a simple RT task (Chocholle, 1940; Snodgrass et al, 1967; Saslow, 1972). The most noticeable exception to this was for the fast-band conditions, and especially for the fast-band data collected in 100-trial blocks. There are several possible reasons for this. First, the length of training on the fast band was very much less than that for the slow band. Second, a few foreperiod time estimations may be included in these fast-band distributions, although actual anticipations, responses before the reaction stimulus, did not occur. Last, all three Ss reported that the 100-trial blocks of fast-band responding were the least interesting and challenging conditions of the experiment. They reported that it was easier to remain alert for conditions in which the responses were delayed or for conditions in which two bands were being switched back and forth.

The results of this experiment imply that the human S can be simultaneously prepared to produce at least two different precise delays, provided that he is first given sufficient training on producing each delay separately.

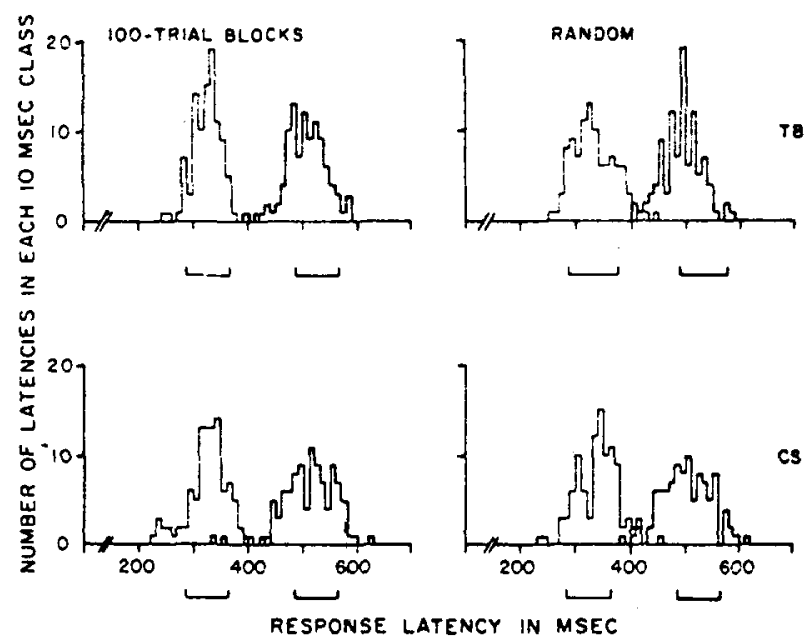

Fig. 3. Response latency histograms for runs with $80-\mathrm{msec}$-wide slow $(285.365 \mathrm{msec})$ and very slow (485-565 msec) payoff bands for two Ss. Data for the left column comes from 100-trial blocks on each band. Data for the right column comes from the first run in which the Ss were randomly switched between bands from trial to trial. Each histogram is composed of 100 latencies. Width and location of the payoff bands is indicated below each histogram. 
One question that this experiment then raises is the role of the foreperiod in determining the Ss' later differential precision timing from the reaction stimulus. In the present experiment, the $S$ had $1,500 \mathrm{msec}$ between receiving the information as to which delay he must produce and timing the reaction.

The fixed warning period of $1,500 \mathrm{msec}$ was selected to ensure that any foreperiod time estimations would noticeably inflate the variability of the latencies to the reaction stimulus. One consequence of the Snodgrass et al (1967) model and its supporting research is that the use of variable foreperiods is no longer obligatory to prevent anticipation of the reaction stimulus. A narrow payoff band combined with a foreperiod of a second or more ensures that attempts to time from the warning stimulus will produce RTs of such high variability that the $S$ will receive very little reinforcement. For example, if for a well-trained $S$ the standard deviation of time estimations is about $10 \%$ of the mean interval being timed (Snodgrass et al, 1967; Saslow, 1972) and the $S$ is asked to produce an RT of $200 \mathrm{msec}$ to a light following a foreperiod of $1,500 \mathrm{msec}$, his RTs timed from the reaction stimulus would have a standard deviation of about $20 \mathrm{msec}$. However, his time estimations would have a standard deviation of about $170 \mathrm{msec}$. Work on monkeys (Saslow, 1972) confirms that a trained animal producing a fast-RT low-variability latency distribution to meet a 50-msec-wide payoff band showed no change in the characteristics of its latency distributions when switched from fixed to randomly varying foreperiods. However, use of variable foreperiods during the training of a monkey to work on any particular band prevented establishment of a low-variability RT distribution, although it did not interfere with performance on the same band once the animal was trained. Therefore, in the present research, fixed foreperiods were used in combination with narrow payoff bands to control anticipation, rather than the more traditional variable foreperiods.

Providing the information as to which delay is required ahead of the reaction stimulus raises several questions. One obvious one is how much this interval could be shortened without affecting the S's ability to precisely produce the different delays. Of course, shortening it would also decrease the variability of foreperiod time estimations and increase the difficulty of detecting inclusion of time estimations in the RT distributions by examination of variability. The question of how the S "sets" himself to produce the different delays is also open. The Ss in the present experiment reported that any attempt to overtly set themselves during the foreperiod disrupted their performance. The only successful approach was to maintain a high degree of altertness and to focus on making the reinforcement light come on. The similarity to the $\mathrm{S}$ of successful trials, whether slow or fast, seemed much greater than any difference in the aftereffects of having responded in one band or the other.
Snodgrass et al (1967) failed to get two different latency distributions when the information as to which payoff band would be in effect on any given trial was contained in the reaction stimulus itself. This suggests that it may be necessary on each trial to provide the $S$ some time ahead of the reaction stimulus with information as to which latency to produce. However, examination of the Snodgrass et al (1967) experiments suggests that the particular bands selected and the training procedures used may not have been optimal for producing two separate latency distributions. They used a fast band of $100-200 \mathrm{msec}$ and a slow band of 200-300 msec. Mean RT in their "choice" condition, which had two reaction stimuli and two responses, was $290 \mathrm{msec}$, regardless of which band was in effect. Mean RT in their "recognition" condition, with one response but two reaction stimuli each signaling a different payoff band, was $250 \mathrm{msec}$ for both fast- and slow-band trials. Since additional time seems to be required for recognizing the nature of the reaction stimulus rather than just reacting to it, it is likely that meeting the fast band of $100-200 \mathrm{msec}$ without time estimating the foreperiod was an impossible task for the S. Different results might be obtained if the two bands were, say, 200-300 msec and 300-400 msec. It might even be better to have a greater separation between the two bands to aid discrimination of the two delays. In addition, use of a training procedure similar to the switch-on-reinforcement training in the present experiment might be better than going straight from a blocked condition to random switching of the two reaction stimuli and their associated payoff bands. Finally, the Snodgrass et al experiment involved 10 different conditions, each one of which was run for only 30 trials within any given session. Continuing training under a single condition until the $S$ is producing stable low-variability latency distributions might improve latency discrimination under random-switch çonditions. The question is still open as to whether the $S$ can choose between two different delays at the time of the reaction stimulus or whether he needs the information as to which delay will be required some time before initiating the delay.

The technique for dual payoff-band control of response latency could have a methodological application in any situation in which it is desirable to accurately vary the interval between stimulus and response over relatively wide time spans. In the investigation of neurophysiological control of behavior, for example, it is often important to determine whether a particular electrophysiological event is locked in time to the occurrence of a stimulus or is a precursor of the response, and locked in time to the response. Evarts (1966) correlated the initiation of activation in single pyramidal tract cells in the monkey with the initiation of gross muscle activity preceding an arm movement in an RT task. In order to determine whether or not the single cell activation was time-locked to the response, he 
had to rely on trial-to-trial variation in response latencies which came from a single latency distribution. In a trained animal with a stereotyped arm movement, this trial-to-trial variability is low. spanning $50 \mathrm{msec}$ or so with, of course, very few trials representing the extremes, making it difficult to obtain significant correlations. The present author's original solution to the problem of varying stimulus to response interval by significant amounts was to change the location of a single response latency distribution by daily gradual changes in the payoff-band location. Using this approach, it was established that the initiation of gross muscle activation in the arm remained time-locked to the response even when the response latency was increased by hundreds of milliseconds (Saslow, 1968). However, since it is generally not possible to observe the same single nerve cell over a period of days, establishing this kind of relationship between single cell activity and behavior would require that radical changes in response latency be made within single sessions without altering either stimulus or response characteristics. Using the dual payoff-band technique would permit such investigation.

In summary, human Ss were found to be able to program themselves accurately for at least two different stimulus-to-response intervals at the same time. This finding both raises some theoretical questions about reaction time and time estimation behavior and offers some methodological utility in the investigation of neural control of behavior.

\section{REFERENCES}

Chocholle, R. Variation des temps de réaction auditifs en fonction de l'intensité à diverse fréquences. L'année Psychologique, 1940, 41, 65-124.

Evarts, E. V. Pyramidal tract activity associated with a conditioned hand movement in monkey. Journal of Neurophysiology, 1965, 29, 1011-1027.

Freund, J. E. Mathematical statistics. Englewood Cliffs, N.J: Prentice-Hall, 1962.

Froeberg, S. The relation between the magnitude of stimulus and the time of reaction. Archives of Psychology, 1907, 8, 1-38.

Gordon, I. E. Stimulus probability and simple reaction time. Nature, 1967, 215, 895-896.

Johanson, A. M. The influence of incentive and punishment upon reaction time. Archives of Psychology, 1922, 54, 1-53.

Lit, A., Young, R. H., \& Shaffer, M. Simple time reaction as a function of luminance for various wavelengths. Perception \& Psychophysics, 1971, 10, 397-399.

Sasiow, C. A. Operant control of response latency in monkeys: Evidence for a central explanation. Journal of Experimental Analysis of Behavior, 1968, 11, 89-98.

Saslow, C. A. Behavioral definition of minimal reaction time in monkeys. Journal of the Experimental Analysis of Behavior, 1972, 18, 87-106.

Snodgrass, J. G. Foreperiod effects in simple reaction time. Anticipation or expectancy? Journal of Experimental Psychology Monograph, 1969, 79, No. 3, Part 2, 1-19.

Snodgrass, J. G., Luce, R. D., \& Galanter, E. Some experiments on simple and choice reaction time. Journal of Experimental Psychology, 1967, 75, 1-17.

Thrane, V. C. Sensory and preparatory factors in response latency. III. Pre-knowledge and regularity of stimuli as antecedent variables. Scandinavian Journal of Psychology, $1961,2,30-44$.

Thrane, V. C. Sensory and preparatory factors in response latency. V. Stimulus blanks as regulator of preparatory set. Scandinavian Journal of Psychology, 1962, 3, 1-15.

(Received for publication March 27, 1972; revision received August 31, 1973.) 